\title{
Efeito dos herbicidas imazapir e imazapique na comunidade fitoplanctônica em lavoura de arroz irrigado'
}

\section{Liange Reck ${ }^{2}$, Geovane Boschmann Reimche ${ }^{3}$, Carlucio Roberto Alves ${ }^{2}$, Katiany do Vale Abreu ${ }^{4}$, Maria Angélica Oliveita ${ }^{5}$ \& Sérgio Luiz de Oliveira Machado ${ }^{3}$}

\author{
'Parte de dissertação de mestrado da primeira autora no Programa de Pós-Graduação em Agrobiologia \\ da Universidade Federal de Santa Maria, RS, Brasil. \\ 2 Universidade Estadual do Ceará, Programa de Pós-Graduação em Biotecnologia, Fortaleza, CE, Brasil. \\ liareck@hotmail.com, carlucio.alves@uece. \\ ${ }^{3}$ Universidade Federal de Santa Maria, Departamento de Fitotecnia, Santa Maria, RS, Brasil. \\ geovane reimche@yahoo.com.br, slomachado@yahoo.com.br \\ ${ }^{4}$ Universidade Estadual do Ceará, Programa de Pós-Graduação em Tecnologia, Fortaleza, Ceará, Brasil \\ katianyabreu@yahoo.com.br \\ ${ }^{5}$ Universidade Federal de Santa Maria, Departamento de Biologia, Santa Maria, RS, Brasil. angelcure@gmail.com
}

Recebido em 02.111.2016

Aceito em 14.XI.2018

DOI 10.21826/2446-8231201873307

\begin{abstract}
RESUMO - Diante da toxicidade de herbicidas aos organismos aquáticos, comumente utilizados em lavouras de arroz irrigado, o presente trabalho visou avaliar o efeito toxicológico da mistura herbicida imazapir+imazapique (Kifix ${ }^{\mathbb{E}}$ ) e compostos isolados na comunidade fitoplanctônica utilizando mesocosmos. Os efeitos destes contaminantes foram avaliados por delineamento experimental em blocos ao acaso com esquema fatorial (5 x 3) e quatro repetições, totalizando 16 parcelas amostrais. Este estudo demonstrou pela Principal Curva de Resposta (PRC) que os herbicidas imazapir+imazapique $\left(\mathrm{Kifix}^{\circledR}\right)$, imazapir e imazapique proporcionam efeito sobre a abundância e composição do fitoplâncton. Os maiores efeitos dos herbicidas em estudo foram encontrados sobre táxons pertencentes às classes Cyanophyceae, Chlamydophyceae e Chlorophyceae. Os tratamentos com herbicidas assemelharam-se ao controle na última coleta, constatando que os herbicidas são mais tóxicos nos primeiros dias após a aplicação.
\end{abstract}

Palavras-chave: algas, imidazolinonas, mesocosmos, Oryza sativa

ABSTRACT - Effect of herbicides imazapyr and imazapicon in the phytoplanktonic community of rice paddy fields. Considering the toxicity of herbicides commonly used in irrigated rice fields to aquatic organisms, this study aimed to assess the toxic effects of the mixture imazapyr+imazapic (Kifix ${ }^{\circledR}$ ), and also of the isolated compounds on the phytoplankton community using experiments in mesocosms. The effects of these contaminants were examined through experimental design with random blocks $(5 \times 3)$ and four repetitions, totaling in 16 sampling units. Using a principal response curves analysis (PRC), the experiment demonstrated that the compound imazapyr + imazapic (Kifix $\left.{ }^{\circledR}\right)$, and the single products imazapyr and imazapic have strong effects on the composition and abundance of phytoplankton communities. The greatest effects were found on three classes of microalgae Cyanophyceae, Chlamydophyceae and Chlorophyceae. The communities treated with the herbicides closely resembled the control blocks on the last sampling day, showing that the toxic effects are more pronounced within the first days of application.

Keywords: algae, imidazolinones, mesocosmos, Oryza sativa

\section{INTRODUÇÃO}

Lavouras de arroz irrigado apresentam características de zonas úmidas, semelhantes a áreas naturais, com mosaico de habitats temporários (Lawler 2001). Estes agroecossistemas têm uma limnologia complexa, caracterizada por alterações químicas, físicas e biológicas aceleradas, no entanto, essa heterogeneidade em escala temporal é um fator que contribui para uma rica biodiversidade (Bambaradeniya et al. 2004).

O uso de herbicidas em lavouras de arroz é potencialmente impactante sobre os organismos aquáticos através da aplicação direta e pelo escoamento superficial (Richards \& Baker 1993). Análises químicas da água realizadas em vários países da Europa indicaram que misturas de agroquímicos estão contaminando águas superficiais (Müller et al. 2002, Irace-Guigand et al. 2004, Chèvre et al. 2006).

Trabalhos demonstram os efeitos de herbicidas utilizados em lavoura de arroz sobre a comunidade fitoplanctônica. Marques et al. (2009) verificaram redução geral na qualidade da água bem como diminuição do crescimento da microalga Pseudokirchneriella subcapitata em área orizícola. Além do mais, foi observado que Clorophyceae são mais afetada no agroecossistema arroz durante seu ciclo com testes com os herbicidas clomazone e quincloraque (Figueiredo et al. 2012). Reimche et al. (2014) constataram que os herbicidas imazetapir e imazapique, grupo químico das imidazolinonas, afetaram a comunidade zooplanctônica com aumento na densidade de cladóceros e copépodes, também em lavouras de arroz. 
Apesar do impacto direto e indireto destes xenobióticos sobre organismos aquáticos (Hanazato 2001) informações disponíveis sobre os efeitos nas comunidades planctônicas em lavoura de arroz são limitadas e o grupo químico das imidazolinonas é amplamente utilizado em Santa Catarina e Rio Grande do Sul para o controle do arroz vermelho. As imidazolinonas, em conjunto com as sulfoniluréias, triazolopirimidina e pirimidilo-oxi-benzoato inibem a enzima acetolactato sintase (ALS), acarretando a inibição da síntese proteica, que, por sua vez, interfere na síntese do DNA e crescimento celular. A inibição do crescimento da planta é devida à escassez de cadeia ramificada e aminoácidos ou por acumulação de substâncias tóxicas intermediárias na síntese da cadeia ramificada (Rizzardi et al. 2002).

$\mathrm{O}$ monitoramento de agroquímicos em ambientes aquáticos, através de bioindicadores químicos, físicos e biológicos é uma ferramenta útil, para averiguar o grau de toxicidade de herbicidas utilizados em lavouras de arroz para conhecimento e acompanhamento dos estados de conservação destes agroecossistemas (Costa et al. 2008). A utilização de microalgas em avaliação ecotoxicológica de ambientes aquáticos é altamente recomendada, pois elas ocupam posições iniciais na cadeia alimentar. Dessa forma, quaisquer alterações na dinâmica de suas populações originam transformação dos níveis tróficos superiores (Klaine et al. 2003). Vidotti \& Rollemberg (2004) ressaltam que a utilização do fitoplâncton como indicador do estresse ambiental é estimável, principalmente, pelo ciclo curto de vida destes organismos, retratando resultados em pequeno espaço de tempo em várias gerações.

Diante do efeito residual dos herbicidas usados em lavoura de arroz e da consequente contaminação de corpos d'água e organismos não-alvo, objetivou-se neste estudo avaliar a toxicidade da mistura herbicida imazapir+imazapique $\left(\right.$ Kifix $\left.^{\circledR}\right)\left(75+25\right.$ g e.a. $\left.\mathrm{L}^{-1}\right)$ e seus compostos isolados na comunidade fitoplanctônica em lavoura de arroz irrigado.

\section{MATERIAL E MÉTODOS}

\section{Área de estudo}

O experimento foi conduzido na unidade de mapeamento Terceiro, localizado no município de São Sepé (S 30¹0'06" W 53 $\left.22^{\prime} 01^{\prime \prime}\right)$, Rio Grande do Sul, Brasil, durante a safra agrícola 2011/2012.

\section{Delineamento experimental e caracterização dos tratamentos}

O delineamento experimental foi de blocos ao acaso em esquema fatorial $(5 \times 3)$ e quatro repetições, totalizando 16 parcelas amostrais. $\mathrm{O}$ fator $\mathrm{A}$ foi composto pelas épocas de coleta da água e do fitoplâncton $\left(1^{\circ}\right.$ - um dia após a aplicação dos herbicidas, $13^{\circ}$ - pleno perfilhamento, $50^{\circ}$ na diferenciação da panícula, $84^{\circ}$ - extrusão da panícula e $113^{\circ}$ - pré-colheita do arroz; antes da drenagem da água) e o fator $\mathrm{B}$ foi à aplicação dos herbicidas compostos pelas misturas formuladas: IM+IZ - imazapir+imazapique $\left(\right.$ Kifix $\left.^{\circledR}\right)\left(75+25\right.$ g e.a. $\left.\mathrm{L}^{-1}\right)$ (concentração indicada a campo em lavouras de arroz irrigado), IM - imazapir, IZ - imazapique, ambos nas mesmas concentrações indicadas a campo em lavouras de arroz irrigado, e CO - tratamento controle (sem uso de herbicida).

$\mathrm{O}$ experimento foi inteiramente casualizado conduzido em parcelas amostrais $\left(5 \mathrm{X} 3 \mathrm{~m}=15 \mathrm{~m}^{2}\right)$, os mesocosmos, isoladas por taipas rondas, possuindo canais em uma das laterais com finalidade de evitar contaminação entre parcelas. A distância entre as parcelas foi de $150 \mathrm{~cm}$ e dentro da parcela foi deixado um espaço de $200 \mathrm{~cm}$ ao entorno das mesmas a fim de evitar sombreamento ao longo do experimento. O controle de plantas daninhas ao longo do experimento foi por meio de capina e arranque manual.

\section{Instalação e condução do experimento}

A cultivar reagente utilizada foi Puitá Inta $\mathrm{CL}^{\circledR}$ (ciclo 120 dias). A semeadura foi realizada na segunda quinzena de novembro de 2011, com semeadura no sistema plantio direto. A adubação e demais tratos culturais foram de acordo recomendação técnica para a cultura (Reunião Técnica da Cultura do Arroz Irrigado 2010), sendo a data de adubação NPK em $45^{\circ}$ dias após a aplicação dos herbicidas.

As aplicações dos herbicidas foram realizadas com pulverizador costal propelido a $\mathrm{CO}_{2}$ (pressão de $40 \mathrm{lbs}$ poll $^{-2}$ ), utilizando-se uma barra com quatro pontas de pulverização em leque XR Teejet 110.01 espaçadas $0,50 \mathrm{~m}$, com volume de calda ajustado para $110 \mathrm{Lha}^{-1}$. As demais técnicas culturais foram realizadas de forma a minimizar a interferência de fatores externos ao experimento.

A irrigação foi iniciada na data da aplicação dos herbicidas (20/12/2011), sendo que a lâmina de água nas parcelas foi monitorada diariamente, mantendo-se a altura de $0,10 \mathrm{~m}$, de acordo com o desenvolvimento da cultura. A condução da água foi provinda de açude e alcançava a área experimental por gravidade. A água foi retirada do canal de superfície através de mangueiras, abastecendo cada parcela individualmente, com a finalidade de evitar contaminação entre parcelas.

\section{Amostragem}

Os procedimentos para coleta, conservação, transporte e análise foram realizados de acordo com APHA (1998). As coletas de água ocorreram mensalmente sempre no período da manhã entre os meses de dezembro e abril. Durante as amostragens, as coletas foram realizadas em altas temperaturas e índices pluviométricos abaixo do esperado para todas as coletas devido à predominância do fenômeno La Niña (fenômeno responsável por passagens rápidas de frentes frias sobre a região sul, com tendência de diminuição da precipitação nos meses de setembro a fevereiro, principalmente no Rio Grande do Sul) presente durante este estudo experimental.

As datas de coleta foram: 21 de dezembro de 2011, 02 de janeiro, 08 de fevereiro, 13 de março e 11 de abril de 2012 , às quais correspondem $1^{\circ}, 13^{\circ}, 50^{\circ}, 84^{\circ}$ e $113^{\circ}$ 
dias após o início da data de irrigação e aplicação dos tratamentos. As coletas foram realizadas nas quatro parcelas com mesmo tratamento perfazendo uma única amostra final, portanto, finalizando com a média de cada tratamento. Foram determinados parâmetros físicos (condutividade elétrica $\mathrm{e}$ turbidez), químicos ( $\mathrm{pH}$, alcalinidade e dureza), nutrientes (nitrato e fosfato) e demanda bioquímica de oxigênio (DBO).

Os procedimentos analíticos e o princípio do método para cada parâmetro foram: condutividade elétrica $(\mathrm{CE})$, medida em $\mu \mathrm{S} \mathrm{cm}^{-1}$, turbidez da água (NTU) e potencial hidrogeniônico $(\mathrm{pH})$ foram medidos por condutivimetria, nefelometria e potenciomatria respectivamente. Os equipamentos utilizados foram condutivímetro (marca HandyLab Scott), turbidímetro (marca PoliControl) e pHmetro digital (marca HandyLab Scott). A dureza total (mg $\mathrm{L}^{-1} \mathrm{de} \mathrm{CaCO}_{3}$ ) foi determinada pelo método de titulometria complexométrica com EDTA. Nitrato $\left(\mathrm{NO}_{3}\right)$ e fosfato $\left(\mathrm{PO}_{4}^{-3}\right)$ foram determinados pelo método de Cromatografia Iônica e a Demanda Bioquímica de Oxigênio (DBO) foi determinada por espectrofotometria de leitura direta em espectrofotômetro HACH DR 2700 com os reagentes NitraVer ${ }^{\mathbb{R}}$ Nitrate $10 \mathrm{~mL}$ e PhosVer ${ }^{\circledR}$ Phosphate $10 \mathrm{~mL}$, respectivamente.

As coletas de fitoplâncton foram realizadas nas mesmas datas de amostragem de água e coletadas diretamente em cada parcela com frascos de $200 \mathrm{~mL}$, cor âmbar, na subsuperfície da água de irrigação, totalizando 80 amostras. As coletas foram realizadas em três pontos diferentes de cada parcela, perfazendo uma única amostra final. As amostras foram fixadas com lugol acético, conforme Bicudo $\&$ Menezes (2006).

\section{Análise das amostras}

Em laboratório, para análise quantitativa, a densidade fitoplanctônica foi estimada segundo a metodologia descrita por Utermöhl (1958), sendo analisadas por microscópio óptico invertido marca Motic AE31. A quantificação de indivíduos (células, colônias, filamentos ou cenóbios) foi efetuada em câmaras de sedimentação com volume de $10 \mathrm{~mL}$ sedimentado por $24 \mathrm{~h}$. A contagem foi realizada por campos aleatórios (Uehlinger, 1964) até atingir 100 indivíduos, de modo que o erro seja inferior a $20 \%$ e o nível de significância na ordem de $95 \%$ (Lund et al. 1958) e com base na curva de rarefação (Bicudo 1990). Para a análise qualitativa foi utilizado microscópio ótico Motic modelo AE21 com câmera digital acoplada. O sistema de classificação adotado foi o de Van den Hoek (1995) e a bibliografia básica para a identificação dos táxons ao nível de gênero foram Franceschini (1992), John et al. (2002), Reviers (2006), Bicudo \& Meneses (2006), Franceschini et al. (2010) e demais trabalhos complementares.

Verificaram-se os parâmetros físicos, químicos, nutrientes e DBO atendem às condições e padrões de qualidade da água estabelecidos na Resolução 357 de 17 de março de 2005 do CONAMA para águas doces, classe 3 (águas podem ser destinadas: ao abastecimento para consumo humano, após tratamento convencional ou avançado, a irrigação de culturas arbóreas, cerealíferas e forrageiras, a pesca amadora, a recreação de contato secundário e a dessedentação de animais). Esta Resolução dispõe sobre a classificação e diretrizes ambientais para o enquadramento dos corpos de água superficiais, bem como estabelece as condições e padrões de lançamento de efluentes.

\section{Análise estatística}

A Principal Curva de Resposta (PRC) foi utilizada para investigar os efeitos dos agroquímicos sobre a comunidade fitoplanctônica. O método PRC é uma técnica multivariada da Análise de Redundância (RDA) que resulta um diagrama mostrando a estrutura dos táxons, entre regimes tratamento e o tratamento controle quando eles progridem com o tempo (Cuppen et al. 2000).

Neste trabalho, as variáveis ambientais utilizadas foram os diferentes agroquímicos e a co-variável foi o tempo, como datas de coleta. O resultado da análise é um diagrama (PRC), no qual o tempo é mostrado no eixo-x e os coeficientes canônicos, relativos ao tratamento controle em questão no eixo-y. Os coeficientes canônicos dos diferentes tratamentos testados são exibidos como desvios do tratamento controle nas respectivas datas de amostragem.

A PRC exibe o peso dos táxons plotado "bk" que pode ser interpretado com a afinidade dos táxons para com a PRC (Mohr et al. 2008). Desta maneira, quanto maior o valor do peso, mais adequada será a resposta do padrão do táxon ao gráfico da PRC, isto é, táxons com pesos de alto valor positivo são indicativos de resposta similar à indicada na PRC, enquanto aqueles com peso de alto valor negativo mostram uma resposta que é oposta a resposta indicada pela PRC.

Os valores de abundância da comunidade fitoplanctônica foram transformados em $\ln (\mathrm{Ax}+1)$ e a significância da PRC foi testada por Monte Carlo usando 999 permutações. Junto a PRC, foram obtidos diagramas com peso dos táxons que é interpretado com a afinidade do táxon a PRC (Mohr et al. 2008). Assim, foram selecionados os táxons com maiores pesos, sendo estes com sinal negativo ou positivo, os quais foram submetidos à análise de variância de medidas repetidas (RM-ANOVA) para testar efeitos da interação tratamento versus tempo. RM-ANOVA foi seguida pela ANOVA univariada para cada data de coleta, a fim de determinar a data exata onde qual tratamento foi significativamente diferente dos demais. Quando a RMANOVA constatou interação ou a ANOVA univariada para uma determinada data foi significativa, o teste de comparação de médias de Tukey $(\mathrm{p}<0,05)$ foi usado para determinar qual tratamento foi mais responsivo para o efeito significativo. Para cada teste, a hipótese de simetria composta dos dados foi verificada com o teste de esfericidade, e quando significativo $(\mathrm{p}<0,05)$, para determinar a significância da interação entre termos do sujeito, foi usado o ajustamento de Greenhouse-Geisser para os graus de liberdade do erro (Dixon 1992).

As análises de PRC foram realizadas através do programa Canoco (Fa. Biometris, v.4.5) (Ter Braak \& Smilauer 1998) e ANOVA-RM e a ANOVA univariada foram realizadas com o programa Statistica (Statsoft Inc. v.8.0). 


\section{RESULTADOS E DISCUSSÃO}

\section{Parâmetros físico-químicos, nutrientes e DBO da água de irrigação}

A turbidez revelou baixos valores ( $>37 \mathrm{NTU}$ ) ao longo do período de amostragem, com exceção do $1^{\circ}$ dia após a aplicação dos herbicidas (DAAH) para imazapir+imazapique $\left(\right.$ Kifix $\left.^{\circledR}\right)$, imazapir e imazapique, em que os dados (Tab. 1) excederam os valores contemplados pelo CONAMA $(\leq 100 \mathrm{NTU})$. Os valores acima de $100 \mathrm{NTU}$ encontrados nos tratamentos imazapir+imazapique $\left(\right.$ Kifix $\left.^{\circledR}\right)$, imazapir e imazapique possivelmente ocorreram pelo material coloidal (herbicidas) suspenso nos mesocosmos, e também, podem estar associados à entrada de água durante a irrigação das parcelas de arroz. A alta concentração de partículas coloidais suspensas na água prejudica a difusão da luz, logo há elevação da temperatura da água e redução de disponibilidade de luz para o fitoplâncton. Desta forma, a turbidez pode atenuar a penetração da luz solar na coluna d'água, reduzindo sua transparência, prejudicando a fotossíntese das algas e plantas aquáticas submersas (Wetzel, 2001).
$\mathrm{O}$ pH demonstrou pouca variação ao longo dos períodos de coleta com média de 5,9 entre os tratamentos. O valor mínimo foi encontrado no $13^{\circ}$ DAAH correspondente a 2,8 (Tab. 1) no tratamento imazapir divergindo os padrões contemplados pelo CONAMA, entre 6 e 9; já o valor máximo foi encontrado no $1^{\circ} \mathrm{DAAH}$ na testemunha com $\mathrm{pH}$ igual a 7,2 (Tab. 1). O baixo valor de $\mathrm{pH} 2,8$ possivelmente influencia a estrutura das comunidades de algas, pois a dinâmica ideal para crescimento do fitoplâncton depende da variação do pH entre 7 e 8,5 (Kubitza 2009), podendo, desta maneira, ser intolerável para algumas espécies, selecionando organismos aquáticos pela acidez do ambiente.

A partir do $84^{\circ} \mathrm{DAAH}$ todos os tratamentos apresentaram registros maiores que o valor de referência do CONAMA para $\mathrm{DBO}\left(\leq 5 \mathrm{mg} \mathrm{L}^{-1}\right)$, com exceção do imazapir no $113^{\circ}$ DAAH (Tab. 1). As amostragens anteriores a $84^{\circ} \mathrm{DAAH}$ (Tab. 1) obtiveram valores abaixo à $5 \mathrm{mg} \mathrm{L}^{-1}$ para todos tratamentos. Possivelmente o aumento da DBO ocorreu pelas descargas de matéria orgânica (adubação NPK que ocorreu cinco dias antes a $\left.50^{\circ} \mathrm{DAAH}\right)$ e o baixo volume de água, já que não ocorreu presença de chuvas marcantes

Tabela 1. Valores das variáveis físicas, químicas, nutrientes e DBO na água de arroz nos tratamentos com aplicação de herbicidas em cinco coletas realizadas na safra agrícola 2011/12. $\mathrm{CO}=$ controle; $\mathrm{KI}=$ imazapir+imazapique $\left(\mathrm{Kifix} \mathrm{x}^{\circledR}\right)$; $\mathrm{IM}=$ imazapir; $\mathrm{IZ}=$ imazapique. $\mathrm{CE}=$ condutividade elétrica; TURB=turbidez; $\mathrm{ALC}=$ alcalinidade; $\mathrm{DUR}=$ dureza; $\mathrm{NIT}=$ nitrato; $\mathrm{FOS}=$ fosfato; $\mathrm{DBO}=$ demanda bioquímica de oxigênio. *Referência CONAMA não contemplados.

\begin{tabular}{|c|c|c|c|c|c|c|c|c|c|}
\hline & & \multicolumn{8}{|c|}{ Parâmetros } \\
\hline & & $\begin{array}{c}\text { C. E. } \\
\left(\mu \text { S.cm }{ }^{-1}\right)\end{array}$ & $\begin{array}{l}\text { TURB } \\
\text { (NTU) }\end{array}$ & $\begin{array}{c}\mathrm{pH} \\
-\end{array}$ & $\begin{array}{c}\mathrm{AlC} \\
\left(\mathrm{mg} \mathrm{L}^{-1}\right)\end{array}$ & $\begin{array}{c}\text { DUR } \\
\left(\mathrm{mg} \mathrm{L}^{-1}\right)\end{array}$ & $\begin{array}{c}\text { NIT } \\
\left(\mu \mathrm{g} \mathrm{L}^{-1}\right)\end{array}$ & $\begin{array}{c}\text { FOS } \\
\left(\mu \mathrm{g} \mathrm{L}^{-1}\right)\end{array}$ & $\begin{array}{c}\text { DBO } \\
\left(\mathrm{mg} \mathrm{L}^{-1}\right)\end{array}$ \\
\hline & & \multicolumn{8}{|c|}{ LIMITE CONAMA } \\
\hline Herbicidas & Datas & - & $\leq 100$ & $6-9$ & - & - & 10000 & 10000 & 5 \\
\hline \multirow{5}{*}{$\mathrm{CO}$} & $1^{\circ}$ & 56,5 & 5,9 & 7,2 & 18,6 & 17,3 & 0,4 & 8,6 & 2,5 \\
\hline & $13^{\circ}$ & 61,3 & 31,2 & 6,8 & 25,3 & 17 & 1,6 & 27,1 & 3,5 \\
\hline & $50^{\circ}$ & 51 & 36,9 & 6,1 & 36,4 & 15,6 & 124 & 124 & 2,3 \\
\hline & $84^{\circ}$ & 41,6 & 29,4 & 6 & 29,7 & 16,5 & 2,3 & 16,8 & $21^{*}$ \\
\hline & $113^{\circ}$ & 41,9 & 12,3 & $5,9^{*}$ & 26,9 & 15,9 & 2,1 & 12,9 & $6^{*}$ \\
\hline \multirow{5}{*}{$\mathrm{IM}+\mathrm{IZ}$} & $1^{\circ}$ & 87,2 & $107^{*}$ & $5,9^{*}$ & 0,3 & 23,1 & 6,3 & 28,2 & 2,8 \\
\hline & $13^{\circ}$ & 124 & 5,7 & $5,3^{*}$ & 34,6 & 18,4 & 2,8 & 15,6 & 4 \\
\hline & $50^{\circ}$ & 50,9 & 10,8 & 6,3 & 29,3 & 14,4 & 36,2 & 117 & 4,7 \\
\hline & $84^{\circ}$ & 45,5 & 10,6 & 6,1 & 24,7 & 23,7 & 6,9 & 15,7 & $7 *$ \\
\hline & $113^{\circ}$ & 39,6 & 14,6 & 6 & 21,6 & 17,5 & 4,4 & 11,3 & $8^{*}$ \\
\hline \multirow{5}{*}{ IM } & $1^{\circ}$ & 97,8 & $119^{*}$ & 6 & 9,6 & 26,1 & 8,4 & 37,1 & 2,7 \\
\hline & $13^{\circ}$ & 68,5 & 9,9 & $2,8^{*}$ & 22,3 & 11,7 & 2,7 & 15,6 & 2 \\
\hline & $50^{\circ}$ & 38,6 & 11,8 & 6,2 & 43,9 & 14 & 34,9 & 105 & 3,9 \\
\hline & $84^{\circ}$ & 43,2 & 12,4 & 6,2 & 23,8 & 23,9 & 7,2 & 16,3 & $9^{*}$ \\
\hline & $113^{\circ}$ & 38,4 & 14,4 & 6,2 & 23,5 & 16,9 & 4,6 & 12,6 & 3,5 \\
\hline \multirow{5}{*}{ IZ } & $1^{\circ}$ & 147 & $124^{*}$ & $5,7^{*}$ & 7,6 & 37,9 & 14,4 & 34,7 & 2,5 \\
\hline & $13^{\circ}$ & 78,2 & 8,7 & $5,4^{*}$ & 23,6 & 12,6 & 1,4 & 15,2 & 2,5 \\
\hline & $50^{\circ}$ & 51,2 & 10,8 & 6,3 & 39 & 11 & 10 & 270 & 4,1 \\
\hline & $84^{\circ}$ & 44,2 & 11,5 & 6,1 & 24,3 & 24,2 & 8,9 & 17,8 & $8^{*}$ \\
\hline & $113^{\circ}$ & 38,3 & 15,3 & 6,1 & 24,4 & 16,3 & 3,9 & 12,5 & $6^{*}$ \\
\hline
\end{tabular}


e predominou escassez de água pluviométrica nas últimas duas coletas do período experimental. Corroborando, Arcova \& Cicco (1999) encontraram relação entre estas variáveis, destacando maior DBO na água proveniente de bacias próxima a áreas agrícolas. Molozzi et al. (2006) verificaram aumento da demanda química de oxigênio (DQO) no mesmo período, dias após a aplicação de adubação NPK.

Os parâmetros condutividade elétrica, alcalinidade, dureza e nutrientes (nitrato e fosfato) atenderam à Resolução 357 de 17 de março de 2005 do CONAMA para águas doces, classe 3 .

\section{Fitoplâncton}

No total de 80 amostras verificadas neste estudo, foram identificados 60 gêneros distribuídos em nove classes, 23 famílias e oito classes: Chlorophyceae (33,33\%), Zygnemaphyceae (23,33\%), Bacillariophyceae (16,7\%), Cyanophyceae (10\%), Euglenophyceae (5\%), Chlamydophyceae $(3,33 \%)$, Oedogoniophyceae $(3,33 \%)$ e Cryptophyceae (1,6\%).

O diagrama de Curva de Resposta Principal - PRC (Fig. 1) da comunidade fitoplanctônica mostrou o efeito dos herbicidas na água de irrigação nas datas de coleta $1^{\circ}, 13^{\circ}$, $50^{\circ}, 84^{\circ}$ e $113^{\circ} \mathrm{DAAH}$. Do total da variância apresentada, no eixo horizontal, $30 \%$ é explicada pelas datas de coleta e $20,9 \%$ pelos tratamentos, já no primeiro eixo do diagrama explicam-se pela variância dos tratamentos químicos em 23,5\% e o teste de permutação de Monte Carlo é altamente significativo $(p=0,002)$.
Os maiores efeitos foram encontrados sobre as populações pertencentes aos gêneros Trachelomonas, Scenedesmus, Spirogyra e Anabaena que apresentaram um alto peso positivo na PRC (Fig. 1) contrastando, de maneira distinta, aumento destas algas na PRC nos tratamentos com os herbicidas imazapir+imazapique $\left(\mathrm{Kifix}^{\circledR}\right)$, imazapir e imazapique, em relação ao controle. Por outro lado, as populações pertencentes aos gêneros Aphanocapsa, Eudorina, Pandorina e Sphaerocystis possuíram peso negativo (Fig. 1) indicando redução destes táxons na PRC para os mesmos herbicidas em relação ao controle, ou seja, são gêneros mais suscetíveis aos xenobióticos em estudo. Suárez-Serrano et al. (2010) também constataram esse aumento e diminuição de densidade de espécies em ambientes em que não foram detectados herbicidas por métodos analíticos, entretanto demonstraram através de métodos biológicos. Os táxons com valores de bk menores que 1.0 e - 1.0 não foram apontados no diagrama, por não apresentarem significância de abundância.

Após a PRC, realizou-se análise de variância, de forma isolada, para os indivíduos de maiores pesos (bk $>2.5$ ou $<-2.5)$, demonstrado que Aphanocapsa, Eudorina, Pandorina e Sphaerocystis obtiveram flutuações médias e temporais entre os tratamentos não significativas $(p>0,05)$ (Fig. 2A-D). Por outro lado, a ANOVA-RM mostrou diferença significativa $(\mathrm{p} \leq 0,05)$ para datas de coleta, média dos tratamentos e interação entre tratamentos para Trachelomonas, Scenedesmus, Spirogyra e Anabaena (Fig. 3A-D). Dessa maneira, para estes gêneros, foi realizada
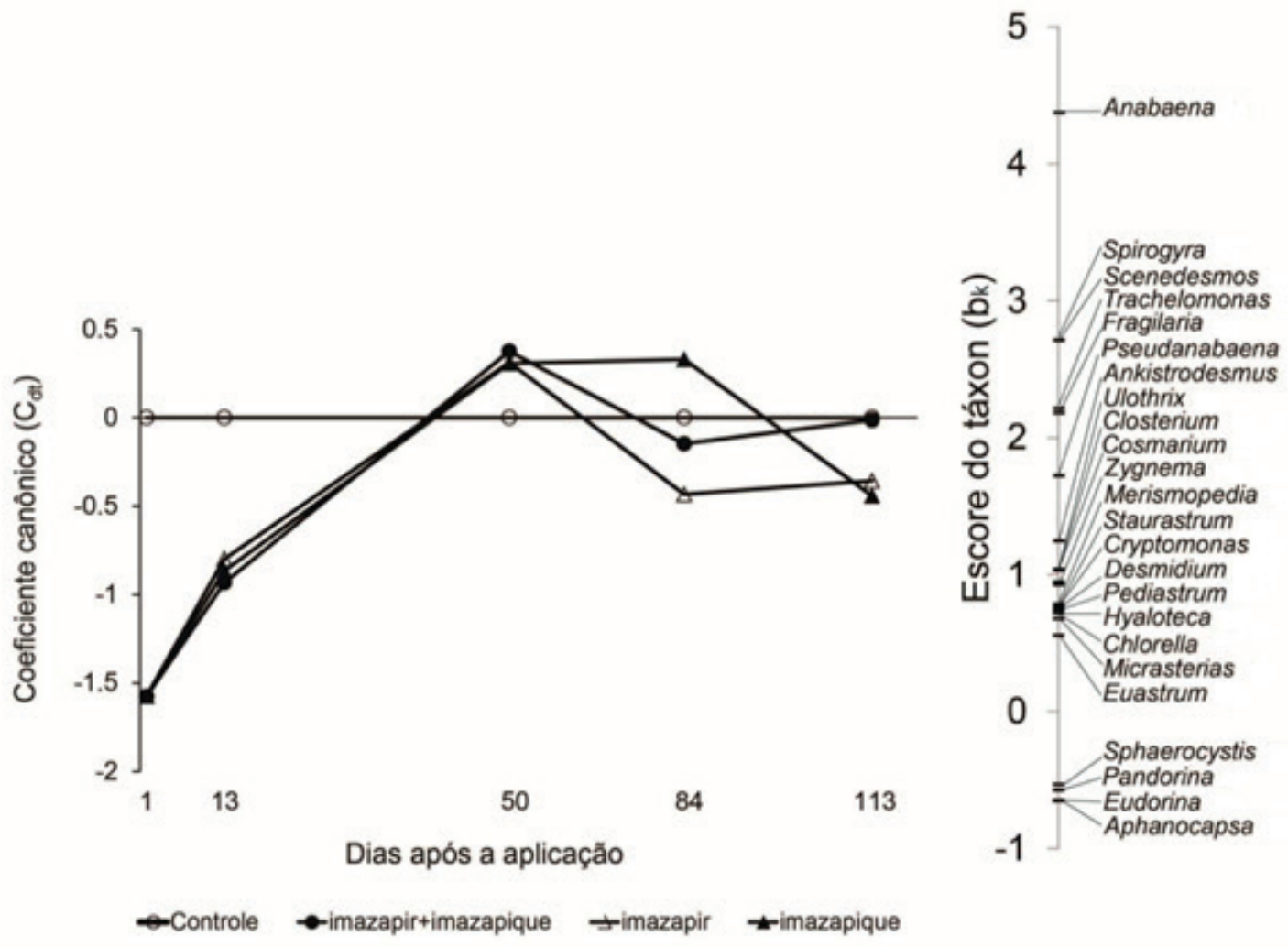

Fig. 1. Principal curva de resposta (PRC) da comunidade fitoplanctônica indicando o efeito dos herbicidas na água de arroz irrigado na safra 2011/12, São Sepé, RS. 

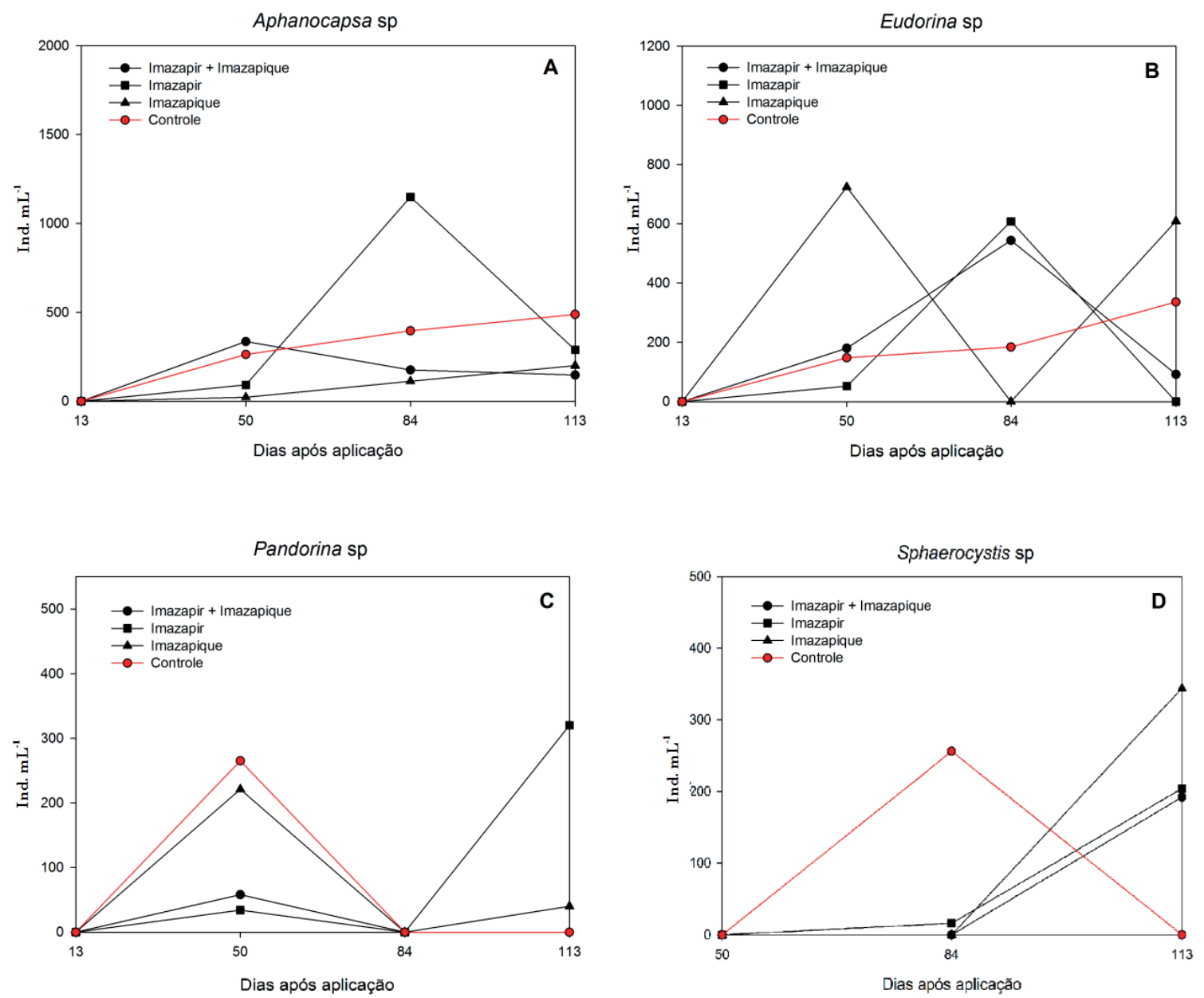

Fig. 2A-D. Dinâmica dos gêneros com maiores valores negativos Aphanocapsa (A), Eudorina (B), Pandorina (C), Sphaerocystis (D) na PRC. As densidades em ind. $\mathrm{mL}^{-1}$ foram calculadas com relação à diferença de densidade no controle durante o período de tempo após a aplicação dos tratamentos com herbicidas.

ANOVA univariada para determinar efeito significativo dos tratamentos, utilizando o teste de comparação de médias de Tukey $(\mathrm{p}<0,05)($ Fig, 3).

Trachelomonas (Fig. 3A) e Scenedesmus (Fig. 3B) não apresentaram densidades em cel.mL ${ }^{-1}$ no $1^{\circ} \mathrm{DAAH}$ para os tratamentos com herbicidas. Por outro lado, no controle obteve-se uma intensa densidade em cel. $\mathrm{mL}^{-1}$. Acredita-se que estes gêneros, primeiramente, foram susceptíveis a mistura herbicida imazapir+imazapique $\left(\right.$ Kifix $\left.^{\circledR}\right)$ e seus compostos isolados. Entretanto, com o passar dos dias devido à dissipação de herbicidas, Trachelomonas e Scenedesmus ampliaram sua tolerância aos herbicidas e podem ter metabolizado os contaminantes em suas células o que explica o crescimento rápido destes dois táxons em poucos dias (entre $1^{\circ}$ e $\left.13^{\circ} \mathrm{DAAH}\right)$. Estudos com mecânismos de estrutura de espécies e composição genética em lavoura de arroz são demonstrados por Kasai \& Hanazato (1995), Kasai (1999) demonstrando a tolerância de espécies em lavoura de arroz.

Os maiores efeitos dos herbicidas imazapir+imazapique $\left(\right.$ Kifix $\left.^{\circledR}\right)$ e seus compostos isolados sobre o fitoplâncton na $\mathrm{PRC}$ foram encontrados sobre aos táxons pertencentes às classes: Cyanophyceae, Chlamydophyceae e Chlorophyceae. Desta forma, percebe-se que os taxa de peso positivo apresentam diferenças expressivas, principalmente, entre o controle e os tratamentos, sobretudo até o $13^{\circ} \mathrm{DAAH}$, confirmando os resultados de Sartori et al. (2011).

Algas verdes têm sido reportadas como sensíveis aos herbicidas linuron, atrazine e metsulfuron metil (WendtRasch et al. 2003, Daam et al. 2009, Pannard et al. 2009). Testes de fitotoxicidade em algas foram realizados com imazetapir, grupo químico das imidazolinonas, apresentando baixa toxicidade para algas verdes e cianobactérias (Peterson et al. 1994, Nyström et al. 1999, Netherland et al. 2009). Em consonância, Jianyi et al. (2002) verificaram em pesquisas toxicológicas com 40 herbicidas em algas verdes, que herbicidas do grupo químico das imidazolinonas são mais tóxicos para o fitoplâncton que herbicidas mimetizadores da auxina.

Os resultados demonstraram diferença significativa nos maiores valores de densidade para todos os gêneros de peso positivo no tratamento controle no $1^{\circ} \mathrm{DAAH}$, em relação aos valores observados nos tratamentos com herbicidas, que não apresentaram concentrações celulares. Logo é possível 
Trachelomonas sp

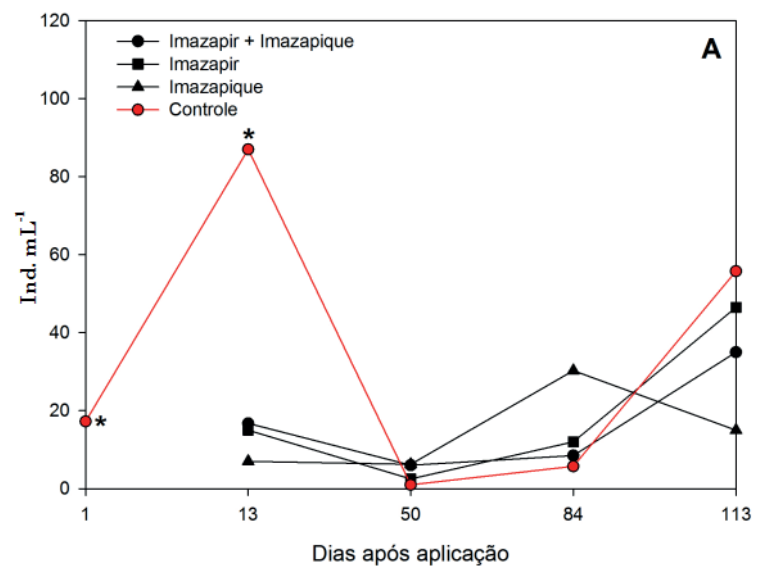

Spirogyra sp

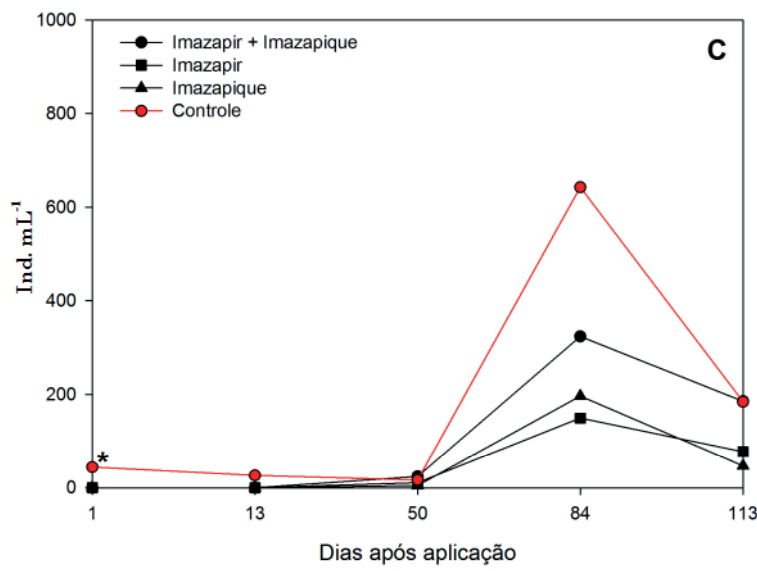

Scenedesmus sp

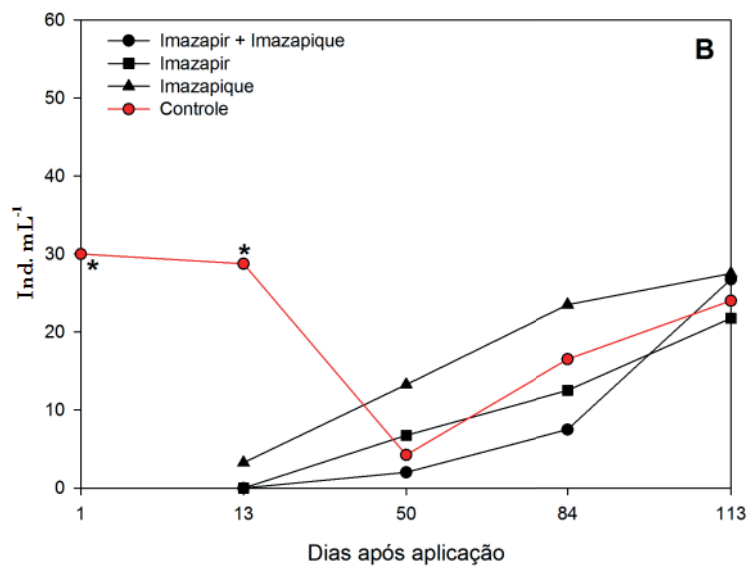

Anabaena sp

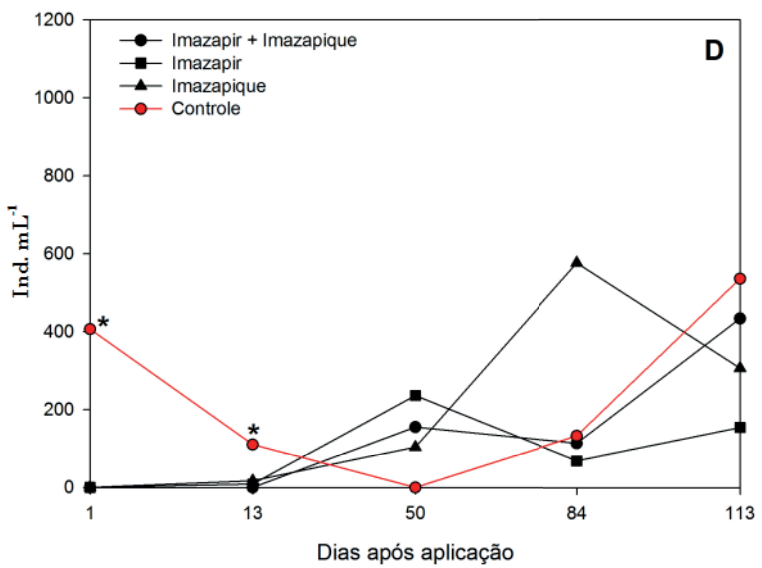

Fig. 3A-D. Dinâmica dos gêneros com maiores valores positivos Trachelomonas (A), Scenedesmus (B), Spirogyra (C), Anabaena (D) na PRC. As densidades em cel. $\mathrm{mL}^{-1}$ foram calculadas com relação à diferença de densidade no controle durante o período de tempo após a aplicação dos tratamentos com herbicidas. *ANOVA univariada para determinar efeito significativo dos tratamentos, utilizando o teste de comparação de médias de Tukey a nível $5 \%$ de probabilidade. $50^{\circ}, 84^{\circ}$ e $113^{\circ}$ DAAH não apresentaram diferença significativa $(\mathrm{p}<0,05)$.

perceber a suscetívidade destes organismos, comprovando a toxicidade de imazapir+imazapique $\left(\mathrm{Kifix}^{\circledR}\right)$ e compostos isolados $24 \mathrm{~h}$ após aplicação dos mesmos. Também, foi possível constatar que no $13^{\circ} \mathrm{DAAH}$ a densidade celular no tratamento controle foi superior àquelas nos tratamentos com herbicidas, provavelmente pelo efeito de imazapir e imazapique retratando diferenças significativas para Trachelomonas, Scenedesmus e Anabaena (Fig. 3A-B-D).

Embora, Trachelomonas, Scenedesmus, Spirogyra e Anabaena não tenham recuperado os níveis celulares no $50^{\circ}$ DAAH para os tratamentos com herbicidas, acreditase ter ocorrido pressão de pastejo, já que o controle também apresentou baixa densidade. Todas as amostras apresentaram ocorrência de zooplâncton, apesar de não identificadas e quantificadas. Já, a parir do $84^{\circ} \mathrm{DAAH}$ percebe-se uma recuperação destes gêneros, sendo que no $113^{\circ} \mathrm{DAAH}$ há uma aproximação expressiva entre os tratamentos para estes organismos em relação ao controle. Assim sendo, percebe-se que o fitoplâncton manteve uma dinâmica semelhante entre os tratamentos testados.
A aproximação na densidade das microalgas $(p>0,05)$ entre os tratamentos e o controle no final do experimento pode estar associada à dissipação dos herbicidas utilizados apresentando menor residualidade e consequentemente gerando menos efeitos sobre as algas, corroborando com os resultados de Knauert et al. (2009) que constataram recuperação do fitoplâncton na segunda semana de estudo em mesocosmos para o herbicida diuron.

Os herbicidas exercem pressão de seleção quando a exposição atinge um determinado nível, durante um período suficiente de tempo variando com as condições ambientais (Morin et al. 2009). O tempo de exposição das microalgas aos herbicidas pode resultar no rápido desenvolvimento de linhagens promovendo o aumento ou diminuição de densidade de espécies tolerantes conforme Kasai (1999) em experimento com diatomáceas e algas verdes.

Pardos et al. (1998) relatam a dificuldade de distinguir possíveis relações, causas e efeitos de misturas de herbicidas, na qual podem ocorrer comportamentos sinérgicos e antagonistas ou aditivos, acarretando efeitos 
nocivos para vários organismos. Ressalta-se que existem variações interespecíficas na suscetibilidade das algas a diferentes herbicidas, logo é indispensável constatar a mistura herbicida imazapir+imazapique $\left(\right.$ Kifix $\left.^{\circledR}\right)$, e também, dos compostos isolados a fim de compreender a dinâmica desta combinação de herbicidas em estudo no fitoplâncton.

Embora sejam fundamentais práticas sustentáveis no uso de herbicidas, pouco se sabe a respeito do destino e impacto destes compostos em ambientes de água doce em clima tropical e subtropical (Castillo et al. 1997, Racke 2003). Com a escassez de resultados nestas regiões, a avaliação depende dos dados de toxicidade de clima temperado (Karlsson 2004, Kwok et al. 2007). Essa diferença de temperatura, umidade, luz solar, entre outros fatores abióticos foi reportada por Daam et al. (2009) que constataram diferenças entre os climas. Os autores verificaram que alguns macroinvertebrados foram mais sensíveis a fungicidas (carbendazim) no clima tropical que em clima temperado.

As diferenças geográficas, a estrutura e funcionamento do ecossistema de água doce e os efeitos de substâncias químicas são distintas entre clima tropical e temperado (Lacher\&Goldstein 1997, Brock et al. 2006) devido a influência de fatores e elementos climáticos. Deste modo, são fundamentais pesquisas voltadas para confronto de resultados entre estes climas, ainda que não haja evidência nas diferenças de sensibilidade entre as espécies na variação do clima (Maltby et al. 2005) para herbicidas.

É possível acreditar que a mistura herbicida imazapir+imazapique (Kifix ${ }^{\circledR}$ ) e seus compostos isolados afetam a comunidade fitoplanctônica diretamente e de forma seletiva perante os testes de toxicidade in situ dos herbicidas nas concentrações recomendadas pelo fabricante, para este estudo. De qualquer forma, trabalhos futuros deverão abordar ambientes mais próximos de sistemas naturais para cumprir um amplo perfil de toxicidade e potencializar riscos ambientais, a fim de estabelecer relações causa-efeito de contaminantes de maneira a considerar outros organismos da cadeia trófica (Wendt-Rasch et al. 2004). Gradativamente, está mais usual técnicas para avaliação da contaminação de sedimentos em bioensaios in situ, os quais consistem em expor diferentes organismos teste no próprio ambiente (Meregalli et al. 2000, Dornfeld et al. 2006, Pérez 2007, Vieira 2010, Reinch et al. 2014) avaliando as respostas reais do funcionamento da área de estudo em questão.

A mistura herbicida imazapir+imazapique $\left(\mathrm{Kifix}^{\circledR}\right)$ pode apresentar efeito residual acentuado utilizando-os consecutivamente, pois o inverno do Rio Grande do Sul apresenta características de baixa incidência luminosa e áreas alagadas sendo inexequível a dissipação destes herbicidas já que é necessário altas temperaturas e $\mathrm{pH}$ ameno para sua dissipação (Loux \& Reese 1993). Desta maneira, acreditase na possibilidade de seleção de organismos em lavouras de arroz em longo prazo, sendo necessários estudos em um período mínimo de duas safras. Nesse sentido, há uma necessidade de maiores informações quanto à toxicidade e persistência da mistura herbicida imazapir+imazapique $\left(\right.$ Kifix $\left.^{\circledR}\right)$ demonstrando a taxa de dissipação destes compostos além de correlacionar com áreas hídricas (lagos e rios) que servem de drenagem para lavouras de arroz.

No sul do Brasil, principalmente Rio Grande do Sul, existe carência de informações para a maioria dos herbicidas utilizados em lavoura de arroz irrigado, sendo, portanto, fundamental estabelecimento de indicadores de impacto ambiental para os ecossistemas locais. Além disso, os indicadores podem servir de subsídio para programas de monitoramento de bacias hidrográficas que recebem o aporte de água de lavouras de arroz. Do mesmo modo, vale ressaltar que atualmente o Brasil é o maior consumidor de agrotóxicos do mundo (Cassal et al. 2014, Pignati et al. 2017), nesse sentido, reforçamos a necessidade da realização de novas pesquisas, a fim de ampliar o diagnóstico para outros herbicidas registrados para a cultura.

\section{CONCLUSÃO}

A adição da mistura herbicida imazapir+imazapique $\left(\right.$ Kifix $\left.^{\circledR}\right)$ e seus compostos isolados provocaram alterações na densidade da comunidade fitoplanctônica em lavoura de arroz irrigado nos primeiros dias após a aplicação destes herbicidas, havendo recuperação dos táxons após 113 dias, no final do experimento.

\section{AGRADECIMENTOS}

À Coordenação de Aperfeiçoamento de Pessoal De Nível Superior (CAPES) à bolsa de mestrado concedida à primeira autora para o desenvolvimento do projeto.

\section{REFERÊNCIAS}

American Public Health Association - APHA. 1998. Standard methods for the examination of water and wastewater. American Public Health Association, Washington. 1569p.

Arcova, F.C.S. \& Cicco, V. 1999. Qualidade da água de microbacias com diferentes usos do solo na região de cunha, estado de São Paulo. Scientia Florestalis 56: 125-134.

Bambaradeniya, C.N.B., Edirisinghe, J.P., de Silva, D.N., Gunatilleke, C.V.S., Ranawana, K.B. \& Wijekoon, S. 2004. Biodiversity associated with an irrigated rice agro-ecosystem in Sri Lanka. Biodiversity and Conservation 13: 1715-1753.

Bicudo, D.C. 1990. Considerações sobre metodologias de contagem de algas do perifíton. Acta Limnologica Brasiliensia 3: 459-475.

Bicudo, C.E.M. \& Menezes, M. 2006. Gêneros de algas de águas continentais do Brasil: Chave para identificação e descrições. RiMa Editora, São Carlos. 502 p.

Brasil, Resolução CONAMA n³57, de 17 de março de 2005. Classificação de águas, doces, salobras e salinas do Território Nacional. Publicado no D.O.U.

Brock, T.C.M., Arts, G.H.P., Maltby, L. \& Van den Brink, P.J. 2006. Aquatic risks of pesticides, ecological protection goals, and common aims in european union legislation. Integrated Environmental Assessment and Management 2:20-46.

Cassal, V.B., Azevedo, L.F., Ferreira, R.P., Silva , D.G. \& Simão, R.S. 2014. Agrotóxicos: uma revisão de suas consequências para a saúde pública. Revista Eletrônica em Gestão, Educação e Tecnologia Digital 18(1): 437-445.

Castillo, L.E., Cruz, E. \& Ruepert, C. 1997. Ecotoxicology and pesticides in tropical aquatic ecosystems of Central America. Environmental Toxicology and Chemistry 16:41-51. 
Chèvre, N., Loepfe, C., Singer, H., Stamm, C., Fenner, K. \& Escher, B.I. 2006. Including mixtures in the determination of water quality criteria for herbicides in surface water. Environmental Science Technology 40: 426-435.

Costa, C.R.,Olivi, P.,Botta, C.M.R. \& Espindola, E.L.G. 2008. A toxicidade em ambientes aquáticos: discussão e métodos de avaliação. Química Nova 31(7):1820-1830.

Cuppen, J.G.M, Brink, P.J., Camps, E., Uil, K.F. \& Brock, T.C.M. 2000. Impact of the fungicide carbendazim in freshwater microcosms. I. Water quality, breakdown of particulate organic matter and responses of macroinvertebrates. Aquatic Toxicology 48: 233-250.

Daam, M.A., Rodrigues, A.M.F., Van den Brink, P.J. \& Nogueira, A.J.A. 2009. Ecological effects of the herbicide linuron in tropical freshwater microcosms. Ecotoxicology and Environmental Safety 72:410-423.

Dixon, W.J. 1992. BMDP Statistical Software Manual. Berkeley. University of California Press, Los Angeles. 251 p.

Dornfeld,C.B., Espíndola, E.L.G., R. Fracácio, R., Rodrigues, B.K. \& Novelli, A. 2006. Comparação de Bioensaios Laboratoriais e "in situ" Utilizando Chironomus xanthus na Avaliação da Toxicidade de Sedimentos do Rio Monjolinho (São Carlos, SP). Jornal of the Brazilian Society of Toxicology 1(2): 161-165.

Figueiredo, M.S., Da Luz, D.S., Cassol, A.P.V. \& Oliveira, M.A. 2012. Avaliação dos efeitos dos herbicidas clomazone e quincloraque sobre a comunidade de algas em cultura de arroz irrigado. In Anais do Simpósio de Ensino e Extensão. Anais da Universidade Francisca de Santa Maria, Rio Grande do Sul. p.1-10.

Franceschini, I.M. 1992. Algues deau douce de Porto Alegre, Brésil (lesDiatomopHyceésexcludes). Biblioteca Phycologica. Berlin, Stwttgart.

Franceschini, I.M., Burliga, A.L., Reviers, B., Prado, J.F. \& Rezig, S.H. 2010. Algas: uma abordagem filogenética, taxonômica e ecológica. Editora Artmed, Porto Alegre. 332p.

Hanazato, T. 2001. Pesticide effects on freshwater zooplankton: an ecological perspective. Environmental Pollution 112(1): 1-10.

Irace-Guigand, S., Aaron, J.J., Scribe, P. \& Barcelo, D. 2004. Acomparison of the environmental impact of pesticide multiresidues and their occurrence in river waters surveyed by liquid chromatography coupled in tandem with UV diode array detection and mass spectrometry. Chemosphere 55: 973-981.

Jianyi, M., Ligen, X., Shufeng, W., Rongquan, Z., Shuihu, J., Songqui, H. \&Youjun, H. 2002. Toxicity of 40 herbicides to the green alga Chlorella vulgaris. Ecotoxicology and Environmental Safety 51:128-132.

John, D.M., Whitton, B.A. \& Brook, A.J. 2002. The Freshwater Algal Flora of the British Isles: An Identification Guide to Freshwater and Terrestrial Algae. University of Cambridge, Cambridge. 702p.

Kasai, F \& Hanazato, T. 1995. Genetic changes in phytoplankton communities exposed to the herbicide simetryn in outdoor experimental ponds. Archives Environmental Contamination Toxicology 128: 154-160.

Kasai, F. 1999. Shifts in herbicide tolerance in paddy field periphyton following herbicide application. Chemosphere 38:919-931.

Karlsson, S.I. 2004. Institutionalized knowledge challenges in pesticide governance: the end of knowledge and beginning of values in governing globalized environmental issues. International Environmental Agreements: Politics 4:195-213.

Klaine, S.J., Lewis, M.A. \& Knuteson, S.L. 2003. Phitotoxicity. In Handbook of Ecotoxicology (D.J. Hoffman, B.A. Rattner, G.A. Burton \& J. Cairns eds.). Boca Raton, New York. 28p.

Knauert, S., Dawo, U., Hollender, J., Hommem, U. \& Knauer, K. 2009. Effects of photosystem II inhibitors and their mixture on freshwater phytoplankton succession in outdoor mesocosms. Environmental Toxicology and Chemistry 28(4): 836-845.

Kubitza, F. 2009. Produção de tilápias em tanques de terra - estratégias avançadas no manejo. Panorama da aquicultura. Disponível em: https://panoramadaaquicultura.com.br/producao-de-tilapias-emtanques-de-terra-estrategias-avancadas-no-manejo/. Acessado em 01.09.2014.

Kwok, K.W., Leung, K.M., Lui, G.S., Chu, S.V., Lam, P.K., Morritt, D., Maltby, L., Brock, T.C., Van den Brink, P.J., Warne, M.S. \& Crane, M. 2007. Comparison of tropical and temperate freshwater species sensitivities to chemicals: implications for deriving safe extrapolation factors. Integrated Environmental Assessment and Management 3:49-67.

Lacher, T.E. \& Goldstein, M.I. 1997. Tropical ecotoxicology: status and needs. Environmental Toxicology and Chemistry 16:100-111.

Lawler, S.P. 2001. Rice fields as temporary wetlands: a review. Israel Journal Zoology 47: 513-528.

Loux, M.M. \& Reese, K.D. 1993. Effect of soil type and $\mathrm{pH}$ on persistence and carryover of imidazolinone herbicides. Weed Technology 7(2): $452-458$

Lund, J.W.G., Kipling, C. \& Lecren, E.D. 1958. The inverted microscope method of estimating algal number and the statistical basis of estimating by counting. Hydrobiologia 11: 143-170.

Maltby, L., Blake, N., Brock, T.C.M. \& Van den Brink, P.J. 2005. Insecticide species sensitivity distributions: importance of test species selection and relevance to aquatic ecosystems. Environmental Toxicology and Chemistry 24:379-388.

Marques, C.R., Pereira, R., Antunes, S.C., Cachada, A., Duarte, A.C. \& Gonçalves, F. 2009. Estudo in situ do efeito de herbicidas numa microalga. Captar 1(1): 113:126.

Meregalli, G., Vermeulen, A.C. \& Ollevier, F. 2000. The use of chironomidae deformation in an in situ test for sediment toxicity. Ecotoxicology and Environmental Safety 47: 231-238.

Mohr, S.M, Feibicke, R., Berghahn, R., Schmiediche \& Schmidt, R. 2008. Response of planktoncommunities in freshwater pond and streanmesocosms to the herbicide metazachlor. Envionmental Pollution 152(3):530-542.

Molozzi, J., Pinheiro, A. \& Silva, M.R. 2006. Qualidade da água em diferentes estádios de desenvolvimento do arroz irrigado. Pesquisa Agropecuária Brasileira 4(9):1393-1398.

Morin,S., Bottin, M., Mazzella, N., Macary, N., Demas, F., Winterton.P. \& Coste, M. 2009. Linking diatom community structure to pesticide input as evaluated through a spatial contamination potential (Phytopixal): A case study in the Neste river system (South-West France). Aquatic Toxicology 94:28-39.

Müller, K., Bach, M., Hartmann, H., Spiteller, M. \& Frede, H.G., 2002. Point- and nonpoint-source pesticide contamination in the Zwester Ohm catchment, Germany. Journal Environmental Quality 31: 309-318.

Netherland, M.D., Lembi, C.A \& Glomski, L.M. 2009. Potential for selective activity of the ALS inhibitors penoxsulam, bispyribacsodium, and imazamox on algae responsible for harmful blooms. Journal of Aquatic Plant Management 47:147-150.

Nyström, B., Björnsäter, B \& Blanck, H. 1999. Effects of sulfonylurea herbicides on non-target aquatic micro-organisms growth inhibition of micro-algae and short-term inhibition of adenine and thymidine incorporation in periphyton communities. Aquatic Toxicology 47:9-22.

Pannard, A., Rouzic, B.L. \& Binet, F. 2009. Response of phytoplankton community to low-dose atrazine exposure combined with phosphorus fluctuations. Archives of Environmental Contamination and Toxicology 57:50-59.

Pardos, M., Benninghoff, C. \& Thomas, R.L. 1998. Photosynthetic and population growth response of the test alga SelenastrumcapricornutumPrintz to zinc, cadmium and suspended sediment elutriates. Journal of Applied Phycology 10:145-151.

Pérez, G.L., Torremorell, A., Mugni, H., Rodríguez, P., Nascimento, M., Allende, L., Bustingorry, J., Escaray, R., Ferraro, M., Izaguirre, I., Pizarro, H., Bonetto, C., Morris, D.P. \& Zagarese, H. 2007. Effects of the herbicide Roundup on freshwater microbial communities: a mesocosm study. Ecological Applications 17(8): 2310-2322.

Peterson, H.G., Boutin, C., Martin, P.A., Freemark, K.E., Ruecker, N.J. \& Moody, M.J.1994. Aquatic phyto-toxicity of 23 pesticides applied at Expected Environmental Concentrations. Aquatic toxicology 28:275-292.

Pignati, W.A., Lima, F.A.N., Lara, S.S., Correa, M.L.M., Barbosa, J.R., Leão, L.H.C. \& Pignatti, M.G. 2017. Distribuição espacial do uso de agrotóxicos no Brasil: uma ferramenta para a Vigilância em Saúde. Ciência e Saúde Coletiva 22(10): 3281-3293.

Racke, K.D. 2003. What do we know about the fate of pesticides in tropical ecosystems?.ACS Symposium Series 853:96-123.

Reimche, G.B., Machado, S.L.O., Zanella, R., Vicari, M.C., Piccinini, F., Golombieski, J.I. \& Reck, L. 2014. Zooplankton community responses to the mixture of imazethapyr with imazapic and bispyribac- 
sodium herbicides under rice paddy water conditions. Ciência Rural 44 (8): 1392-1397.

Reunião Técnica da Cultura do Arroz Irrigado. 2010. Arroz irrigado: recomendações técnicas da pesquisa para o Sul do Brasil. In $28^{\circ}$ Reunião Técnica da Cultura do Arroz Irrigado, Bento Gonçalves, RS. Sociedade Sul-Brasileira de Arroz Irrigado, Porto Alegre, p. 1-188.

Reviers, B. 2006. Biologia e filogenia das algas. Tradução Iara Maria Franceschini. Porto Alegre: artmed. 280p.

Richards, R.P. \& Baker, D.B. 1993. Pesticide concentration patterns in agricultural drainage networks in the Lake Erie basin. Environmental Toxicology and Chemistry 12:13-26.

Rizzardi, M.A., Vidal, R.A., Fleck, N.G. \& Agostinetto, D. 2002. Resistência de plantas aos herbicidas inibidores da acetolactato sintase. Planta Daninha 20(1): 149-158.

Sartori, G.M.S., Marchesan, E., Luz, D.S., Cassol, A.P.V., Figueiredo, M.C.S., Oliveira, M.A., Silveira, M.V.E. \& Ferreira, R.B. 2011. Manejo da adubação e seus efeitos na ocorrência de algas e na produtividade de arroz irrigado em áreas com residual de imidazolinonas. Ciência Rural 41(8):1323-1330.

Suárez-Serrano, A., Ibãnez, C., Lacorte, S. \& Batata, C. 2010. Ecotoxicological effects of rice field waters on selected planktonic species: comparison between conventional and organic farming. Ecotoxicology 19:1523-1535.

Ter Braak, C.J.F. \& Smilauer, P. 1998. CANOCO reference manual and user"s guide to canoco for windows. Software for canonical community ordination (version 4). Micro Computer Power, Ithaca, New York, USA.

Uehlinger, V. 1964. Etude statistique des methods de denobrement planctonique. Archaeological Science 17(2):121-123.

Utermöhl, H. 1958. ZurVervollkommnung der quantitativen PhytoplanktonMethodik.Mitteilungen der internationalenVereinigung der theoretische und angewandte. Limnology 5:567-596.

Van den Hoek, C., Mann, D.G. \& Jahns, H.M. 1995. Algae. An Introduction to Phycology. Cambridge University Press, Cambridge. 623p.

Vidotti, E.C. \& Rollemberg, M.C. 2004. Algas: da economia nos ambientes aquáticos à bioremediação e à química analítica. Química Nova 27 (1):139-145.

Vieira, B.H. 2010. Efeitos do agrotóxico Vertimec ${ }^{\circledR}$ no fitoplâncton: um estudo laboratorial e "in situ”. Dissertação 181 f., Universidade Federal de São Carlos, São Paulo.

Wendt-Rasch, L., Pirzadeh, P. \& Woin, P. 2003. Effects of metsulfuron methyl and cypermethrin exposure on freshwater model ecosystems. Aquatic Toxicology 63:243-256.

Wendt-Rasch, L., Van den Brink, P.J., Crum, S.J. \& Woin, P. 2004.The effects of a pesticide mixture on aquatic ecosystems differing in trophic status: responses of the macrophyteMyriophyllumspicatum and the periphytic algal community. Ecotoxicology and Environmental Safety 57:383-398

Wetzel, R. G. 2001. Limnology: Lake and River Ecosystems. Academic Press, San Diego.1006 p. 